\title{
ENERGY-MOMENTUM RELATIONSHIP IN InAs †
}

\author{
G. H. Parker and C. A. Mead \\ California Institute of Technology, Pasadena, California 91109
}

(Received 8 July 1968)

\begin{abstract}
We have performed surface-barrier tunneling measurements on $p$-type InAs and found the energy dependence of the wave vector in the forbidden gap to be in agreement with Franz's relationship based on a two-band model.
\end{abstract}

In 1965 , Lewicki and co-workers ${ }^{1-3}$ showed that it was possible to determine energy dependence of the imaginary part of the electron wave vector in the forbidden gap of an insulator or semiconductor by measurements of tunneling current as a function of thickness and applied voltage. Later in an elegant series of experiments Padovani and Stratton ${ }^{4}$ applied a similar technique to Schottky barriers on GaAs, a material which has subsequently been studied by other workers. ${ }^{5,6}$ Since both the thickness of the depletion layer and the energy of the final state depend upon applied voltage, they were able to characterize a considerable portion of the energy gap with a simple voltage-current measurement on a single sample.

InAs is an ideal material for study by this technique $^{7}$ because the barrier energy on $p$-type material is nearly equal to the band gap ${ }^{8}$ and hence the entire forbidden region is accessible. In addition, the conduction- and valence-band effective masses are nearly equal, and the energy gap small; therefore, the two-band model ${ }^{9}$ should be an excellent approximation to the actual energy-wave-vector relationship in the forbidden gap. ${ }^{10}$ Our results indicate that this is indeed the case.

Samples were prepared by two techniques: (a) Crystals were cleaved in air quickly placed on a metal mask in a Vac-Ion system. The chamber was evacuated to approximately $10^{-1}$ Torr and metal dots evaporated on the cleaved surface through the mask. The typical sample was exposed to air less than 30 sec prior to pumpdown.

(b) Crystals were cleaved in vacuum in an evaporating stream of metal. After completion of the evaporation, the samples were removed from the vacuum, masked with either black wax (for single dots) or photoresist (for guard-ring structures) and the remaining metal etched away.

No systematic differences were found between samples formed by the two techniques. A summary of the sample characteristics are given in Table I. Typical forward current-voltage curves taken with the samples immersed in liquid nitrogen are shown in Fig. 1.

Although in principle a semiconductor may be characterized over a substantial energy range by tunneling measurements on a single sample, in practice the range of accurate data obtained is limited at very low current levels by leakage and at high forward bias by series resistance. For this reason it is necessary to use material of high carrier concentration (and hence large tunneling current) for measurements at low biases. With material of somewhat lower concen-

Table I. Characteristics of samples used in the $E-k$ analysis. Sample numbers correspond to those in Figs. 1 and 2 .

\begin{tabular}{cccccc}
\hline \hline Sample & Metal & Preparation & $\begin{array}{c}\varphi \\
(\mathrm{eV})\end{array}$ & $\begin{array}{c}\text { Area } \\
\left(\mathrm{cm}^{2}\right)\end{array}$ & $\begin{array}{c}N \\
\left(10^{17} \mathrm{~cm}^{-3}\right)\end{array}$ \\
\hline 1 & $\mathrm{Au}$ & $\begin{array}{c}\text { Air } \\
\text { cleave } \\
\text { Air } \\
\text { cleave } \\
\text { Air } \\
\text { cleave } \\
\text { Vacuum } \\
\text { cleave } \\
\text { Vacuum } \\
\text { cleave }\end{array}$ & 0.51 & $7.5 \times 10^{-5}$ & 6 \\
5 & $\mathrm{Cu}$ & 0.38 & $7.5 \times 10^{-5}$ & 1.6 & $1.6 \times 10^{-5}$ \\
guard ring & $\mathrm{Al}$ & 0.38 & $1.6 \times 10^{-4}$ & $1.6 \times 10^{-5}$ & 1.6 \\
\hline \hline
\end{tabular}




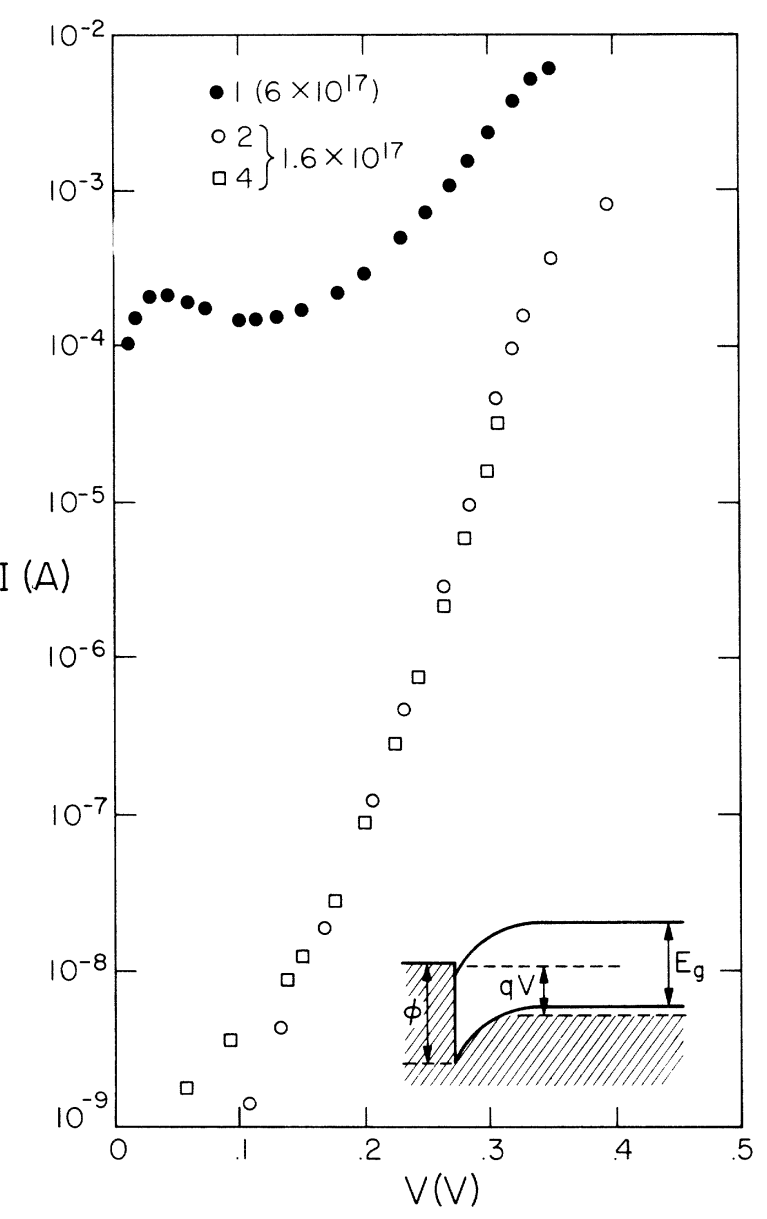

FIG. 1. Typical $77^{\circ} \mathrm{K}$ current-voltage plots for Schottky barriers on $p$-type InAs.

trations, the tunneling current is reduced and considerably higher forward bias may be applied before the current level rises to the point where resistive drop becomes important. Since the InAs surface is highly $n$-type ${ }^{8}$ the effects of surface leakage at low current levels are more severe than with GaAs. This effect and room-temperature blackbody radiation made the data unusable below approximately $10^{-8} \mathrm{~A}$ even in the better samples.

The dependence of the imaginary part of the wave vector $k$ on energy $E$ was determined from the measured current $I$ at applied forward voltage $V$ by the relationship ${ }^{5,11}$

$$
-k^{2}\left(E=\varphi-q I^{\prime}\right)=\left(N q^{2} / 2 \epsilon\right)(\varphi-q V)(d \ln I / d V)^{2} .
$$

Results of this procedure are shown in Fig. 2.

On samples 2-5, the barrier energy $\varphi$ and carrier concentration $N$ were determined from $77^{\circ} \mathrm{K}$ capacitance versus reverse bias voltage using a

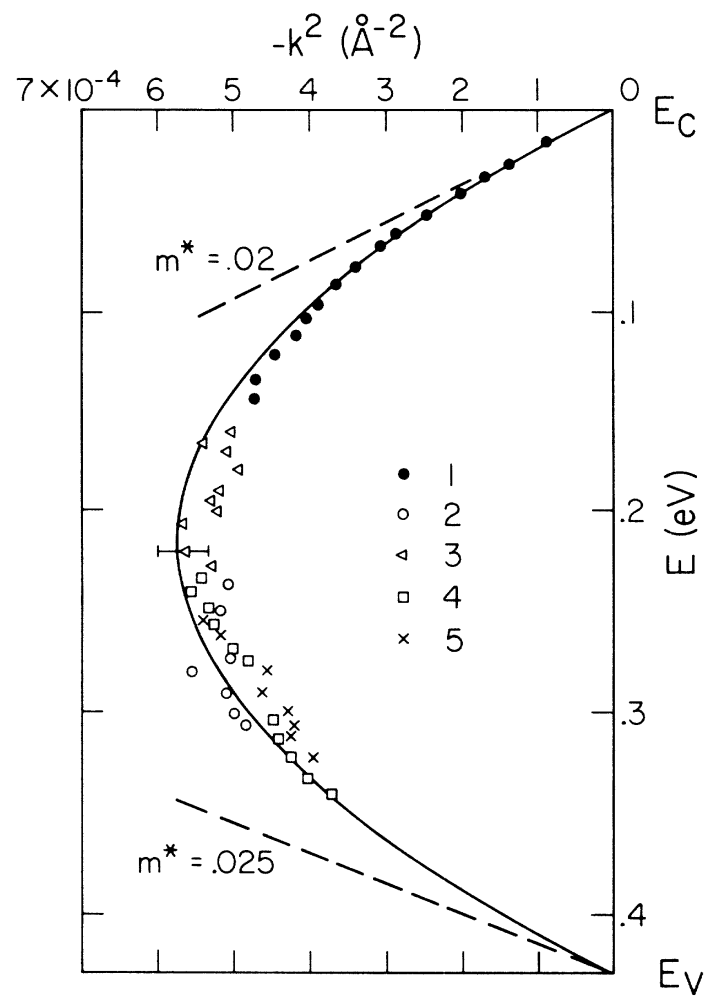

FIG. 2. Energy-wave-vector plot derived from current-voltage data. The solid curve is the two-band expression for $m^{*}=0.02$.

dielectric constant $\epsilon$ of 12.6.

On sample 1 the barrier energy was taken to be the voltage at the current minimum plus the band $\operatorname{gap}^{7}\left(0.43 \mathrm{eV}\right.$ at $\left.77^{\circ} \mathrm{K}\right) .^{10}$ Since the low impedance of this sample precluded measurements of capacitance versus voltage, the carrier concentration was adjusted to bring the $k$ values into agreement with those of the other samples in the region of overlap. This procedure gave a concentration of $6 \times 10^{17}$, in good agreement with Hall measurements supplied by the manufacturer (given as $7 \times 10^{17}$ ).

The solid curve in Fig. 2 is Franz's symmetrical two-band expression ${ }^{2}$

$$
\boldsymbol{E}=\left[\frac{E g^{2}}{4}+\frac{\hbar^{2} k^{2} E g}{2 m^{*}}\right]^{1 / 2}-\frac{E g}{2}
$$

assuming an effective mass $m^{*}$ of 0.02 times the mass of the free election. The reported values for the conduction and valence band are 0.02 and 0.025 , respectively. ${ }^{12}$

We wish to thank François Padovani for pointing out the unique nature of InAs in connection with these experiments. 
$\dagger$ Work supported in part by the Office of Naval Research.

${ }^{1}$ G. W. Lewicki, thesis, California Institute of Technology, 1965 (unpublished).

${ }^{2}$ G. W. Lewicki and C. A. Mead, Phys. Rev. Letters 16, 939 (1966).

${ }^{3}$ R. Stratton, G. W. Lewicki, and C. A. Mead, Solid State Electron. 27, 1599 (1966).

${ }^{4}$ F. A. Padovani and R. Stratton, Phys. Rev. Letters 16,1202 (1966).

${ }^{5}$ J. W. Conley and G. D. Mahan, Phys. Rev. 161, 681 (1967).

${ }^{6}$ M. Millea, M. McColl, and C. A. Mead, to be published.

\footnotetext{
${ }^{7}$ F. Padovani, in "Physics of III-V Compounds" (Academic Press, Inc., New York, to be published).

${ }^{8}$ C. A. Mead and W. G. Spitzer, Phys. Rev. Letters 10,471 (1963).

${ }^{9}$ W. Franz, in Handbuch der Physik, edited by S。Flügge (Springer-Verlag, Berlin, Germany, 1956), Vol. 18 , p. 155 .

${ }^{10}$ E. O. Kane, private communication.

${ }^{11}$ Strickly speaking, the semiconductor degeneracy should appear on the right but its omission results in negligible error for the conditions used here.

${ }^{12} \mathrm{C}$. Benoit à la Guillaume, Constantes Selectionées Relatives aux Semiconducteurs (Pergamon Press, S.A.R.L., Paris, France, 1961).
}

\title{
RAMAN SCATTERING BY OPTICAL MODES OF METALS*
}

\author{
D. W. Feldman, James H. Parker, Jr., and M. Ashkin \\ Westinghouse Research Laboratories, Pittsburgh, Pennsylvania 15235 \\ (Received 3 May 1968)
}

\begin{abstract}
Raman scattering from the optical modes of the metals $\mathrm{Be}$ and $\mathrm{AuAl}_{2}$ has been observed. The measurements were carried out using argon-ion laser excitation. One Raman line was observed for each of these metals. A frequency of $455 \mathrm{~cm}^{-1}$ was obtained for $\mathrm{Be}$ and $266 \mathrm{~cm}^{-1}$ for $\mathrm{AuAl}_{2}$. The result for $\mathrm{Be}$ is compared with neutron scattering data.
\end{abstract}

We have observed Raman scattering from the optical modes of the metals $\mathrm{Be}$ and $\mathrm{AuAl}_{2}$. Beryllium is of hcp structure with two atoms per unit cell. The space group for this lattice is $D_{6 h}{ }^{4}$ and there is one $E_{2 g}$ Raman active optical mode. Gold-aluminum has the $\mathrm{CaF}_{2}$ structure and has one $T_{2 \mathrm{~g}}$ Raman active mode.

The Raman scattering was observed using argon-ion laser excitation. The sample was placed within the laser cavity with its polished face acting as a third cavity mirror. This type of sample configuration has been reported previously in application to $\mathrm{Si}$ and $\mathrm{Ge}$. The $\mathrm{AuAl}_{2}$ sample was a single crystal while the Be sample was a polycrystalline ingot with grain size of the order of several millimeters. The surface of both samples were mechanically polished.

One Raman line was observed for each of these materials. Figure 1 shows the Stokes line for Be with 4880-A excitation. The Stokes component has also been observed for Be with $5145-\AA$ excitation. Figure 2 shows the Stokes component for 4880- $\AA$ excitation which was obtained with the $\mathrm{AuAl}_{2}$ sample. The anti-Stokes line for $4880 \AA$ and the Stokes line for $5145 \AA$, respectively, were also observed for $\mathrm{AuAl}_{2}$. It is evident from Figs. 1 and 2 that the Raman lines are superimposed on an appreciable background due to strong scattering of the exciting light from the metal surface. This background is appreciable in comparison with the Raman lines because the Raman scattering is weak on account of the small scattering volume of the metals and because it is difficult to obtain a perfect polish on the metal surfaces. In Fig. 2 the background shown as a dashed line is subtracted from the recorder trace to give the Raman lines shown at the bottom of the figure. Table I gives the observed frequencies and linewidths at $300^{\circ} \mathrm{K}$ for these samples. The Raman intensity of Be was

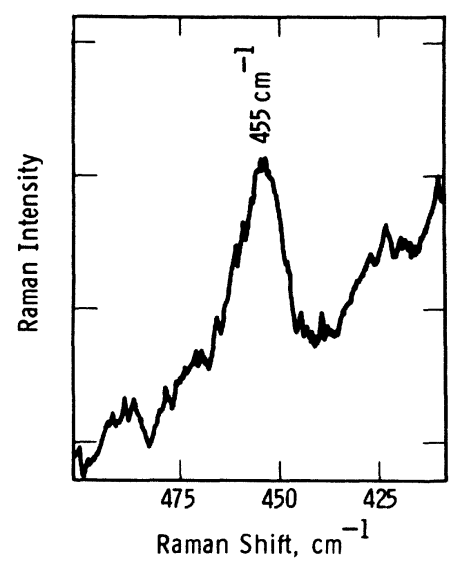

FIG. 1. Observed Raman line for Be metal: Stokes component for $4880-\AA$ excitation. The instrumental resolution was $8 \mathrm{~cm}^{-1}$, the time constant was $300 \mathrm{sec}$, and the scan rate was $1 \mathrm{~cm}^{-1} / \mathrm{min}$. 